\title{
DÉCEMBRE 1976
}

58 A NNÉE - N॰696
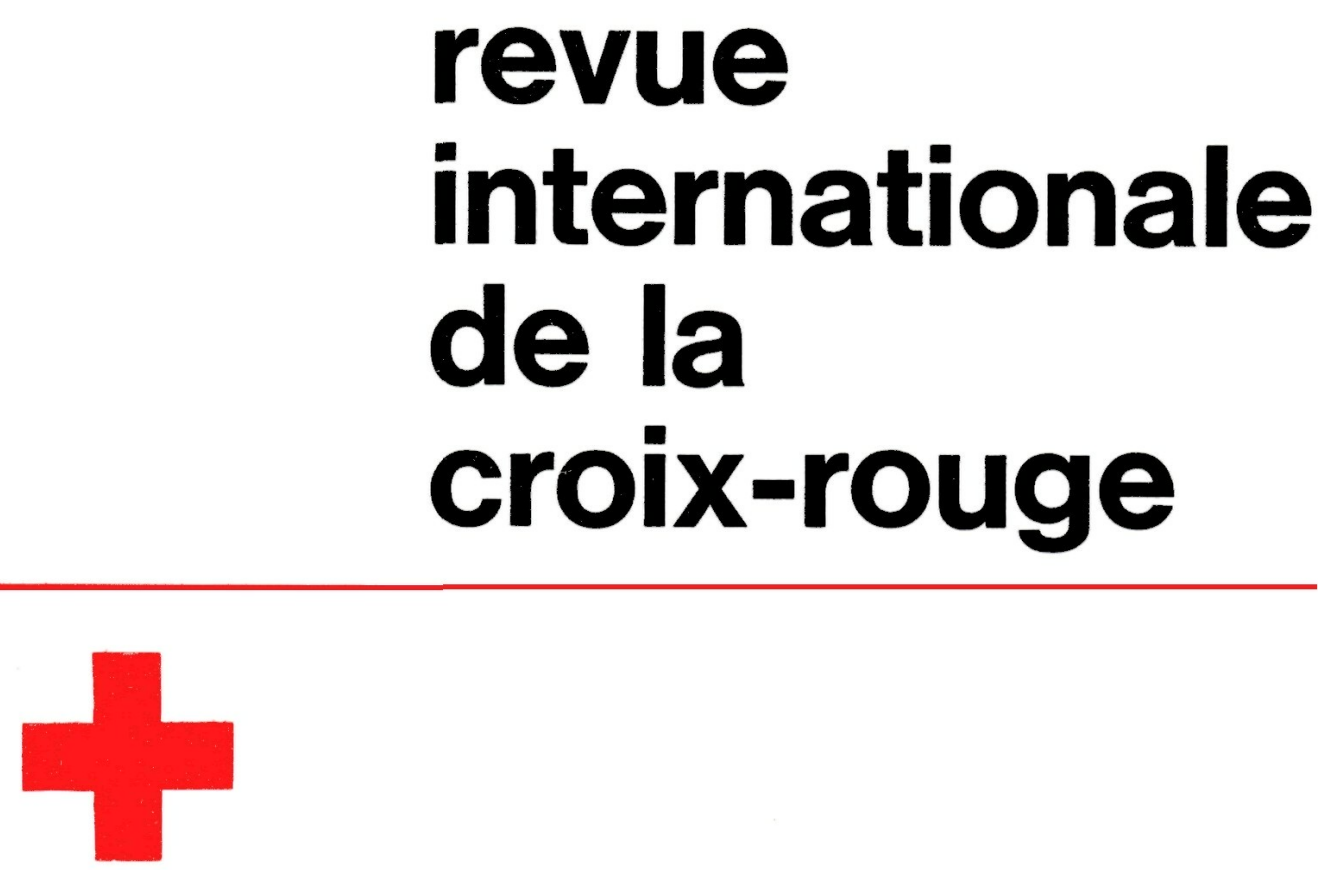

INTER ARMA CARITAS

GENÈVE

COMITÉ INTERNATIONAL DE LA CROIX-ROUGE FONDÉ EN 1863 


\title{
COMITÉ INTERNATIONAL DE LA CROIX-ROUGE
}

MM. ALEXANDRE HAY, avocat, ancien directeur général de la Banque nationale suisse, président (membre depuis 1975)

JEAN PICTET, docteur en droit, président de la Commission juridique, directeur de l'Institut Henry-Dunant, professeur associé à l'Université, Genève, vice-président (1967)

HARALD HƯBER, docteur en droit, juge fédéral, vice-président (1969)

$M^{\text {me }}$ DENISE BINDSCHEDLER-ROBERT, docteur en droit, professeur à l'Institut universitaire de hautes études internationales, Genève, juge à la Cour européenne des droits de l'homme (1967)

MM. MARCEL A. NAVILLE, licencié ès lettres, président du CICR de 1969 à 1973 (1967)

JACQUES F. DE ROUGEMONT, docteur en médecine (1967)

ROGER GALLOPIN, docteur en droit, ancien directeur général du CICR (1967)

WALDEMAR JUCKER, docteur en droit, secrétaire de l'Union syndicale suisse (1967)

VICTOR H. UMBRICHT, docteur en droit, administrateur (1970)

PIERRE MICHELI, licencić en droit, ancien ambassadeur (1971)

GILBERT ETIENNE, professeur à l'Institut universitaire de hautes études internationales et à l'Institut d'études du développement, Genève (1973)

ULRICH MIDDENDORP, docteur en médecine, chef de la clinique chirurgicale de l'Hôpital cantonal, Winterthour (1973)

$M^{\text {me }}$ MARION BOVÉE-ROTHENBACH, diplômée M.S.W. de l'Université de Michigan, maîtreassistant à l'Ecole des sciences sociales et politiques de l'Université, Lausanne (1973)

MM. HANS PETER TSCHUDI, docteur en droit, ancien conseiller fédéral (1973)

HENRY HUGUENIN, directeur de banque (1974)

GOTTFRIED DE SMIT, administrateur (1974)

JAKOB BURCKHARDT, docteur en droit, ministre plénipotentiaire, président du Conseil des Ecoles polytechniques fédérales (1975)

THOMAS FLEINER, docteur en droit, professeur à l'Université de Fribourg (1975)

HERBERT LÜTHY, docteur en philosophie, professeur d'histoire à l'Université de Bâle (1975)

Membres honoraires: M"le LUCIE ODIER, vice-présidente d'honneur; MM. HANS BACHMANN, GUILLAUME BORDIER, PAUL CARRY, M ${ }^{\text {me }}$ MARGUERITE GAUTIER-VAN BERCHEM, MM. ADOLPHE GRAEDEL, EDOUARD DE HALLER, ERIC MARTIN, RODOLFO OLGIATI, MAX PETITPIERRE, PAUL RUEGGER, DIETRICH SCHINDLER, FRÉEERIC SIORDET, ALFREDO VANNOTTI.

\section{CONSEIL EXÉCUTIF}

\author{
M. ROGER GALLOPIN, président \\ M. VICTOR H. UMBRICHT, vice-président \\ $M^{\text {me }}$ DENISE BINDSCHEDLER-ROBERT \\ M. GILBERT ETIENNE \\ $D^{\text {T ULRICH MIDDENDORP }}$ \\ M. JEAN PICTET \\ M. GOTTFRIED DE SMIT \\ M. PIERRE MICHELI, membre suppléant
}




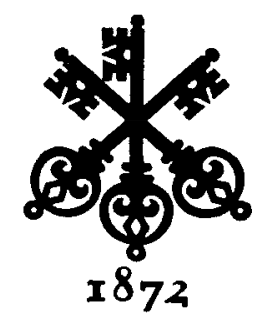

PRÉSENTE SUR LES CINO CONTINENTS,

LA GRANDE BANQUE AUX TROIS CLEFS

EST À VOTRE DISPOSITION POUR EFFECTUER

TOUTES VOS OPÉRATIONS FINANCIËRES

\section{SOCIÉTÉ DE BANQUE SUISSE}

SCHWEIZERISCHER BANKVEREIN - SOCIETA DI BANCA SVIZZERA

SWISS BANK CORPORATION

SIËGE DE GENÈVE

2, RUE DE LA CONFÉdÉRATION

TÉL. 224111

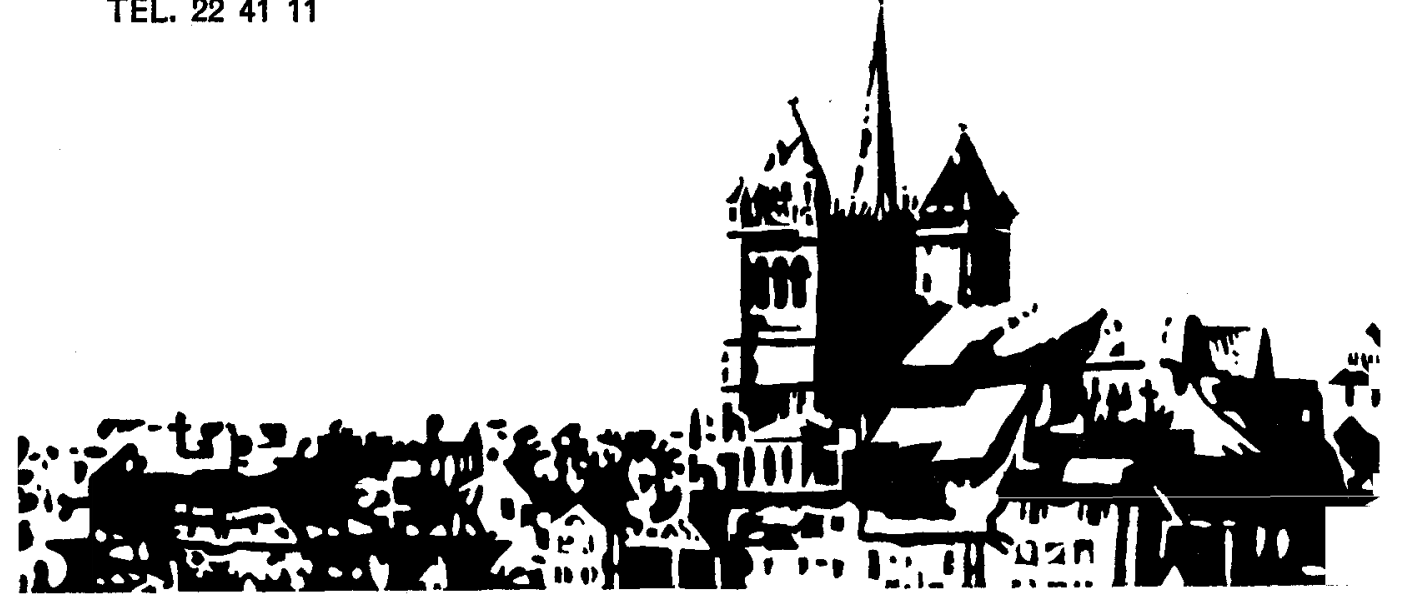




\section{FIDUCHAIRE OFOR S.A.}

Place Saint-Gervais 1, Genève - Tél. 317050

Expertises, révisions et organisations comptables

Domiciliation et administration de sociétés

Assainissements et liquidations

Interventions et conseils en matière fiscale

\section{JOURTAL DE GENEVE}

deux imprimeries

deux adresses

rue François-Dussaud 20

1211 Genève 8 - Tóléphone 431940

rue Général-Dufour 5-7

1211 Genève 11 - Téléphone 280350

TYPO - OFFSET - ROTATIVE 


\section{REVUE INTERNATIONALE DE LA CROIX-ROUGE}

\section{SOMMAIRE}

COMITEE INTERNATIONAL DE LA CROIX-ROUGE

\section{DANS LE MONDE} DE LA CROIX-ROUGE

\section{FAITS ET DOCUMENTS}

\section{DÉCEMBRE 1976 - № 696}

Hans Haug : La Croix-Rouge internationale 697

Le Comité international de la Croix-Rouge et la torture

Activités extérieures :

Afrique - Amérique latine - Asie - MoyenOrient

A Genève:

Présidence du Conseil exécutif . . . . . . . . 723

Participations aux Conventions de Genève . . . 723

Les principes de la Croix-Rouge en langue arabe 724

Une table des matières de l'édition anglaise de la Revue internationale . . . . . . . . 725

Commission permanente de la Croix-Rouge internationale . . . . . . . . . . . . 726

Réévaluation du rôle de la Croix-Rouge . . . . 727

Session extraordinaire du Conseil des Gouverneurs de la Ligue . . . . . . . . . . . . . 728

Viet Nam . . . . . . . . . . . . . . . . 730

Bulgarie . . . . . . . . . . . . 730

Finlande . . . . . . . . . . . . . 731

Une nouvelle adhésion au Protocole de Genève . 732 VII ${ }^{e}$ Congrès de la Société internationale de droit pénal militaire et de droit de la guerre . . . . 732

Qui était le frère d'Henry Dunant ? . . . . . 733

Table des matières 1976 . . . . . . . . . . 734 
INTERNATIONAL

REVIEW

OF THE RED CROSS

\section{REVISTA INTERNACIONAL DE LA CRUZ ROJA}

\section{EXTRAITS}

DE LA REVUE

EN LANGUE ALLEMANDE
Une édition en langue anglaise et une en langue espagnole paraissent chaque mois. Elles sont en principe identiques à l'édition française, et peuvent être obtenues aux mêmes conditions.

Gedenkstein für Eglantyne Jebb - Seminar über das humanitäre Völkerrecht und seine Verbreitungsmethoden - Inhaltsverzeichnis.

Elle est publiée chaque mois par le Comité international de la Croix-Rouge.

17, avenue de la Paix, 1211 Genève (Suisse) - Compte de chèques postaux $12-1767$.

Abonnement un an: Fr. 30,-,; le numéro: Fr. 3,-.

RÉDACTION: J.-G. LOSSIER

Seuls les textes signés par le Comité international de la Croix-Rouge engagent la responsabilité de celui-ci. 